\title{
Pulmonary Complications in Falciparum Malaria in a tertiary care center in costal Andhra Pradesh
}

\author{
Dr. Sahoo A. K., Dr. Das K. K. \\ ${ }^{1,2}$ Asst. Prof., Dept. of General Medicine, Maharajah's Institute of Medical Sciences, Vizianagaram, Andhra \\ Pradesh, India
}

\begin{abstract}
Malaria is a treatable cause of acute lung injury (ALI) and acute respiratory distress syndrome (ARDS). Due to variation in clinical presentation, pulmonary manifestations in malaria has always been underdiagnosed. A total 95 patients with severe falciparum malaria were included in this study. The diagnosis of malaria was confirmed by examination of peripheral smear / OptiMal test/ QBC. Arterial blood gas analysis was done in patients who developed respiratory symptom. It was observed that out of 41 patients showing respiratory symptoms ARDS was present $8((8.42 \%)$ no. of cases, Acute lung injury in $4(4.21 \%)$ cases and Pneumonia in 4 (4.21\%) cases. ARDS was more common in patients with heavy parasitemia $(75 \%$ with $>20 \%$ parasitemia), hypoalbuminemia(75\%) and anemia(62.5\%). All patients with respiratory distress were given ventilator support. Patients with ARDS showed a high mortality rate of 75\%. ARDS in malaria have a poor disease outcome. Early diagnosis, institution of specific antimalarial treatment and assisted ventilation can be life-saving.
\end{abstract}

Key words: Malaria, ARDS, ALI, pulmonary

\section{Introduction}

Malaria, a protozoan disease remains a major health problem worldwide, especially in tropical and subtropical countries. According to WHO the burden of disease in India due to malaria appears to be 20 million cases and 15,000 deaths per annum (1).

Usually patients with uncomplicated malaria present with fever and non-specific symptoms, complicated and severe malaria can cause multi-organ involvement. Lung involvement in malaria is quite common and may range from upper respiratory tract involvement to more fatal complications like acute lung injury (ALI) and acute respiratory distress syndrome (2) (3). Most of these complications are recognised easily and addressed quickly. Previous studies have shown three types of pulmonary manifestations in falciparum malaria namely bronchitic, pneumonic and bronchopneumonic forms (4) (5).

In india, there have been few studies focusing on the pulmonary manifestations of falciparum malaria. We, therefore, undertook to examine the clinical characteristics of pulmonary involvement in patients who had falciparum malaria presented to our hospital.

\section{Objective}

To study the incidence and various clinical presentations of pulmonary involvement in patients with falciparum malaria.

The secondary objective was to determine any possible correlation of these pulmonary manifestations with other clinical parameters, if any.

\section{Materials and Methods}

The patient population included in this study was adult malaria patients aged 18 years and above who had been admitted in medicine wards or attended the outpatient department due to malaria during one year period from April 2010 to March 2011.

The diagnosis of malaria was clinically suspected and later confirmed by examination of thick and thin smear / OptiMal test/ Quantitative Buffy coat (QBC). The peripheral blood films were prepared from prick of finger, stained by conventional Leishman's stain and Geimsa stain seen under oil immersion (100×) taking care to examine particular upper and lower margins and tail end of the film (because the parasites are numerous there) and a minimum of 100 fields were examined before declaring the slides negative for Plasmodium falciparum. Only those cases with asexual forms of Plasmodium falciparum in the blood by smear examination or found positive in OptiMal test for Plasmodium falciparum or QBC positive were included. Malaria patients with renal failure, severe anemia, and volume overload were excluded. The patients with any other systemic disease, chronic respiratory disease, chronic obstructive pulmonary disease, bronchitis, pulmonary tuberculosis and heart failure were excluded from study. Smokers, pregnant or lactating females and those unable to give informed consent were also excluded. 
Anti-malarial treatment was administered according to the standard institutional treatment algorithm. Additional antibiotic course (if superimposed bacterial infection suspected), oxygen administration, mechanical ventilation, hydration and electrolyte replacement were left to the discretion of the treating physicians, although the latest WHO treatment guidelines was encouraged (21).

Data collection: According to standardized procedure, we recorded the following information prospectively: (1) demographics (2) co-morbidities (3) presenting complaints at admission (4) pulmonary manifestations (5) vital signs (6) biochemical profile and events and treatments during the course of treatment in case of hospitalized patients.

The diagnosis of ALI and ARDS were considered according to the American-European Consensus Conference definition published in 1994 (6).

Table 1. Definitions of acute lung injury and acute respiratory distress syndrome

\begin{tabular}{|l|l|}
\hline Acute lung injury & Acute respiratory distress syndrome \\
\hline Acute onset & Acute onset \\
$\mathrm{PaO} 2 / \mathrm{FIO} 2<300$ & $\mathrm{PaO} 2 / \mathrm{FIO} 2<200$ \\
$\mathrm{SpO} 2 / \mathrm{FIO} 2<315$ & $\mathrm{SpO} 2 / \mathrm{FIO} 2<235$ \\
$\mathrm{Bilateral} \mathrm{infiltrates}$ on the frontal chest radiograph & Bilateral infiltrates on the frontal chest radiograph \\
$\mathrm{PCWP}<18 \mathrm{~mm} \mathrm{Hg}$, or no clinical evidence of left & $\begin{array}{l}\mathrm{PCWP}<18 \mathrm{~mm} \mathrm{Hg}, \text { or no clinical evidence of left } \\
\text { atrial hypertension }\end{array}$ \\
\hline
\end{tabular}

Daily monitoring of vital parameters were done during hospital stay. Blood and urine routine examination were done in all patients. PA view chest X-ray was done in all patients who complained of respiratory symptoms or had abnormal chest examination findings. Serum creatinine, urea and random blood sugar were done in all patients to rule out renal involvement and to document hypoglycemia . A 12 lead ECG was done in selected cases.

\section{Results}

A total of 157 potential cases who were hospitalized in the Medicine Department with complain of fever were screened for the study. Of these, 95 patients were found to be eligible, and were included in the study.

56 patients were males $(59 \%)$ and 39 females $(41 \%)$ with a male to female ratio of 1.4:1. This reflects the current epidemiology of malaria in the coastal areas of Andhra Pradesh with male dominance. The maximum number of cases were seen in the age group of 21-49 years which constituted $62 \%(n=35)$ of the total cases. The elderly age group (>60 years) was involved in only $12 \%(n=4)$ cases.

In this study, $65.3 \%$ cases were infected with P. falciparum $(n=62)$ whereas mixed infections $(n=33)$ accounted for $34.7 \%$. More than three-fourth of cases had complicated falciparum malaria $(n=74,78 \%)$. The parasite load in these patients is shown in table no 2.

Table-2

\begin{tabular}{|l|l|l|}
\hline$\%$ of parasitized RBCs & No. & Percentage \\
\hline$<5 \%$ & 32 & $33.68 \%$ \\
\hline $6-10 \%$ & 15 & $15.78 \%$ \\
\hline $11-15 \%$ & 26 & $27.36 \%$ \\
\hline $16-20 \%$ & 16 & $16.84 \%$ \\
\hline$>20 \%$ & 6 & $6.31 \%$ \\
\hline
\end{tabular}

Respiratory symptoms were observed in less than fifty per cent of cases $(n=41,43 \%)$. Cough was the predominant respiratory symptom $(\mathrm{n}=27,28.4 \%)$ with one-fourth had only dry cough. Tachypnoea with a respiratory rate $>30 / \mathrm{min}$ was observed in $27 \%$ of total cases $(n=26)$ whereas a small subset had dyspnoea $(\mathrm{n}=14,14.7 \%)$.

In this study ALI was seen in only 4 patients whereas ARDS was present in 8 patients. Concurrent pneumonia was detected in 4 patients. The radiological evaluation in these patients showed that the patients with ALI and ARDS were associated with diffuse opacities involving the peripheries whereas findings suggestive of lobar consolidation were the predominant radiological features in patients with pneumonia. In all the four cases sputum culture were positive and responded to third generation cephalosporins.

The severe degree of respiratory involvement was seen in patients with heavy parasitemia, low serum albumin and severe anaemia (table no-3).

Table-3 
Pulmonary Complications in Falciparum Malaria in a tertiary care center in costal Andhra Pradesh

\begin{tabular}{|l|l|l|l|l|l|l|}
\hline & $\begin{array}{l}\text { Parasitemia } \\
<20 \%\end{array}$ & $\begin{array}{l}\text { Parasitemia } \\
>20 \%\end{array}$ & $\begin{array}{l}\text { Sr } \\
\text { albumin } \\
<3.5 \mathrm{~g} / \mathrm{dl}\end{array}$ & $\begin{array}{l}\text { Sr } \\
\text { albumin } \\
>3.5 \mathrm{~g} / \mathrm{dl}\end{array}$ & Hb <5g\% & $\mathrm{Hb}>5 \mathrm{~g} \%$ \\
\hline ARDS & $2(25 \%)$ & $6(75 \%)$ & $6(75 \%)$ & $2(25 \%)$ & $5(62.5 \%)$ & $3(37.5 \%)$ \\
\hline $\begin{array}{l}\text { Acute Lung } \\
\text { injury }\end{array}$ & $3(75 \%)$ & $1(25 \%)$ & $1(25 \%)$ & $3(75 \%)$ & $1(25 \%)$ & $3(75 \%)$ \\
\hline
\end{tabular}

\section{Discussion}

The most frequent presentation of malaria is that of a pronounced febrile illness with rigors. However, the clinical features of malaria can be extremely diverse because the parasitized red cell circulates to every organ and tissue within the body and therefore has the potential for producing a wide variety of pathology. In endemic areas the manifestations of severe disease in children are mainly those of cerebral malaria, often with convulsions, respiratory distress and severe anemia, whereas adults are more likely to develop multi-organ failure (e.g. renal failure) and are less likely to have convulsions or severe anemia.

Respiratory symptoms and signs are common in plasmodium falciparum malaria with a frequency of $4 \%-18 \%$ in uncomplicated malaria. In our study respiratory manifestations were found in $33 \%(n=7)$ in uncomplicated malaria which is consistent with earlier studies. A dry cough may be present in $20 \%$ to $50 \%$ of patients with malaria (7) (8) (9) (10). Tachypnea may result from high fever, anemia, and lung involvement. In our study patients with respiratory involvement, $24.2 \%$ had dry cough, $27 \%$ had tachypnoea.

A number of terms have been used to describe respiratory symptoms and signs in clinical series of severe malaria caused by P falciparum. Respiratory distress is commonly used and has many causes, including respiratory compensation for a severe metabolic acidosis, concomitant pneumonia, aspiration pneumonia, fluid overload, ALI/ARDS, and severe anemia (which is more important in young children). In the large South East Asian Quinine Artesunate Malaria Trial (SEAQUAMAT) study of adult severe malaria, 175 patients $(12 \%)$ had respiratory distress (11).

The risk of developing ARDS/pulmonary edema in patients presenting with uncomplicated falciparum malaria is low: $0.1 \%$ (3 of 3,300) in US Army soldiers in Vietnam (12). In severe malaria, reported incidence rates vary widely between $<2 \%$ to about $25 \%$. The wide variation in incidence rates is mainly because of the applied definitions of the ARDS (13) (14) (15). In our study, the respiratory distress was present in $14.7 \%$ cases with an incidence rate of ARDS and ALI in $8.42 \%$ and $4.21 \%$ respectively.

We found that the patients who developed ARDS had anaemia (62\%), hypoalbuminemia (75\%) or heavy parasitemia (75\%). This indicates that the pathogenesis of ALI/ARDS in severe falciparum malaria is multifactorial and includes the effects of sequestration of parasitized erythrocytes, host immunologic reactions to lung-specific sequestration or systemic malaria infection, superimposed pulmonary infections. ARDS has grave prognostic significance. In settings without mechanical ventilation, ARDS mortality was $81 \%$ in Rajasthan, India, and $100 \%$ in Vietnam (13) (14). Even with availability of mechanical ventilation, ARDS accounts for $>95 \%$ (70 of 74) in Krishnan and Karnad ICU series (16). In our study 6 patients died out of 8 patients showing a mortality of $75 \%$.

In severe malaria respiratory symptoms and signs are quite common and therefore, signs and symptoms of pneumonia may be overlooked in the differential diagnosis of respiratory distress in these patients. Both the diseases can be present concomitantly in up to $13 \%$ in those geographical areas where both the illnesses are common (17) (18) . O'Dempsey et al (17) showed that although certain signs like cough, chest wall recession, and crepitations, were more likely in radiographically confirmed pneumonia, none was sufficiently discriminating to exclude malaria. In our study pneumonia was found only in $4 \%$ of cases. In all these cases productive cough, tachycardia and chest pain were the predominant features with radiological evidence of pneumonia.

Anaemia, coagulopathy and ARDS was more common in patients with high degree of parasitemia. Respiratory symptoms were more common and severe in patients in whom parasitemia was more than $10 \%$ in peripheral smear. Though certain degree of correlation exists between the severity of the disease and parasitaemia, but quantification of the asexual parasitaemia does not accurately reflect the parasite load due to erythrocyte adherence and sequestration, the peripheral smears may not reveal the parasite load accurately. ARDS was more common in patients with hemoglobin less than $5 \mathrm{gms} / \mathrm{dl}$ whereas ALI was common in those having hemoglobin more than $5 \mathrm{gms} \%$. In the present study anaemia was seen in $72 \%$ cases which was little less when compared to other studies (14) (19) (20). The principal cause of anemia in falciparum malaria is destruction of parasitized and non-parasitized red blood cells which correlates with severity of infection. Anemia is more common and severe in falciparum malaria because it affects red blood cells of all ages. 


\section{CONCLUSION}

Respiratory distress is more common in patients with heavy parasitemia and hypoalbuminemia. Malaria is an important curable cause of ALI/ARDS and anemia worsens the situation. Therefore, early diagnosis and treatment with good supportive care will lead to better outcome in falciparum malaria.

\section{References}

[1] World Health Organization. World Malaria Report. Geneva:; 2009.

[2] Mohan A, Rao MH, Sharma SK, Reddy MK. Acute respiratory failure requiring assisted ventilation in patients with falciparum malaria. Am J Respir Crit Care Med. 2003; 167: p. A907.

[3] Vijayan VK. Tropical parasitic lung diseases. Indian JChest Dis Allied Sci. 2008; 50: p. 49-66.

[4] Taylor WR, White NJ. Malaria and the lung 2002; 23: 457-68. Clin Chest Med. 2002; 23: p. 467-68.

[5] Taylor WR, Cañon V V, White NJ. Pulmonary manifestations of malaria: recognition and management. Treat Respir Med. 2006; 5: p. 419-28.

[6] Bernard GR, Artigas A, Brigham KL, Carlet J, Falke K, Hudson L, et al. The American-European consensus conference on ARDS: definitions, mechanisms, relevant outcomes and clinical trial coordination. Am J Respir Crit Care Med. 1994; 149: p. 818-24.

[7] Anstey NM, Jacups SP, Cain T, et a. Pulmonary manifestations of uncomplicated falciparum and vivax malaria: cough, small airways obstruction, impaired gas transfer, and increased pulmonary phagocytic activity.. 2002; 185(9): 1326--1. J Infect Dis. 2002; 185(9): p. 1326-1334.

[8] Hovette P, Touze JE, Laroche R. Pulmonary manifestations of malaria [in French]. Bull Soc Pathol Exot. 1990; 83(4): p. 479-486

[9] Rajput R, Singh H, Singh S, Meena, Tiwari UC. Pulmonary manifestations in malaria. J Indian Med Assoc. 2000; 98(10): p. 612614

[10] Gozal D. The incidence of pulmonary manifestations during Plasmodium falciparum malaria in non immune subjects. Trop Med Parasitol. 1992; 43(1): p. 6-8.

[11] Dondrop A, Nosten F, Stepniewska K, Day N, White N. South East Asian Quinine Artesunate Malaria Trial (SEAQUAMAT) group, Artesunate versus quinine for treatment of severe falciparum malaria: a randomised trial. Lancet. 2005; 366(9487): p. 717725 .

[12] Sheehy TW, Reba RC. Complications of falciparum malaria and their treatment. Ann Intern Med. 1967; 66(4): p. 807-809.

[13] Tran TH, Day NP, Nguyen HP, al e. A controlled trial of artemether or quinine in Vietnamese adults with severe falciparum malaria. N Engl J Med. 1996; 335(2): p. 76-83.

[14] Kochar D, Kumawat BL, Karan S, Kochar SK, Agarwal RP. Severe and complicated malaria in Bikaner (Rajasthan), western India. Southeast Asian J Trop Med Public Health. 1997; 28(2): p. 259-267.

[15] Mohan A, Sharma SK, Bollineni S. Acute lung injury and acute respiratory distress syndrome in malaria. J Vector Borne Dis. 2008; 45(3): p. 179-193.

[16] Krishnan A, Karnad DR. Severe falciparum malaria: an important cause of multiple organ failure in Indian intensive care unit patients. Crit Care Med. 2003; 31(9): p. 2278-2284.

[17] O'Dempsey TJ, McArdle TF, Laurence BE, Lamont AC, Todd JE, Greenwood BM. Overlap in the clinical features of pneumonia and malaria in African children. Trans R Soc Trop Med Hyg. 1993; 87(6): p. 662-665.

[18] English M, Punt J, Mwangi I, McHugh K, Marsh K. Clinical overlap between malaria and severe pneumonia in Africa children in hospital. Trans R Soc Trop Med Hyg. 1996; 90(6): p. 658-662.

[19] Sharma SK, Das RK, Das PK. Hematological and coagulation profile in acute falciparum malaria. JAPI. $1992 ; 40:$ p. 581 -83.

[20] Bajiya HN, Kochar DK. Incidence and neurological sequelae in survivors of cerebral malaria. JAPI. 1996; 44: p. 679-81.

[21] WHO guidelines for the treatment of malaria $2^{\text {nd }}$ edition 2010 